\title{
Microscopic nuclear form factors for the pygmy dipole resonance
}

\author{
E. G. Lanza, ${ }^{1,2}$ A. Vitturi, ${ }^{3,4}$ and M. V. Andrés ${ }^{5}$ \\ ${ }^{1}$ INFN Sezione di Catania, Catania, Italy \\ ${ }^{2}$ Dipartimento di Fisica e Astronomia, Universitá di Catania, Italy \\ ${ }^{3}$ Dipartimento di Fisica e Astronomia, Universitá di Padova, Italy \\ ${ }^{4}$ INFN Sezione di Padova, Padova, Italy \\ ${ }^{5}$ Departamento de FAMN, Facultad de Física, Sevilla, Spain
}

(Received 24 March 2015; published 11 May 2015)

\begin{abstract}
It is shown that the use of a proper form factor in the study of the pygmy dipole resonance (PDR) is of paramount importance in order to determine the correct values for the relevant quantities characterizing these new modes. Form factors calculated within a microscopic model are compared with those provided by different macroscopic collective models. Their differences, shown in the shape and magnitude, are reflected on the calculated cross section and therefore jeopardize the extracted physical quantities.
\end{abstract}

DOI: 10.1103/PhysRevC.91.054607

PACS number(s): 25.60.-t, 25.70.-z, 24.30.Gd, 21.60.Ev

\section{INTRODUCTION}

Among the new features revealed in the studies of nuclei far from the stability line, one of the most interesting aspects present in nuclei with neutron excess is the occurrence of a small peak in the low energy region of the dipole strength distribution in almost all nuclei with a very high $N / Z$ ratio $[1,2]$. The importance of these states, interesting by themselves, is enhanced by their relation with the symmetry energy term of the equation of state (EOS) [3-6]. Theoretical as well as experimental studies have shown that for these dipole states an investigation using an isoscalar probe is possible due to the well-established mixture of isoscalar and isovector natures of these states [2,7]. This is due to the distinctive behavior of the neutron and proton transition densities, which are in phase inside the nucleus, while at the surface only the neutron one gives the major contribution, the proton one being almost zero. Therefore, both the isoscalar and isovector transition densities have a peak at the surface region, arising just from the neutron contribution and for this reason having almost the same intensity $[1,2,8,9]$.

In the last few years many experiments have been done with an isoscalar probe to study the pygmy dipole resonance (PDR) states [2,10-16] on stable nuclei. Many more experiments have been already performed or are presently planned with nuclei far from the stability line. In all these cases the extraction of information about the strength and the relative energy-weighted sum rule (EWSR) percentage is mostly based on distorted-wave Born approximation (DWBA) calculations which depend very much on the models used for the inelastic form factor. Therefore the use of proper form factors is of paramount importance in the deduction of these physical quantities. In this paper, different form factors are compared and their differences in the corresponding differential cross section are illustrated. These form factors are calculated within the double-folding procedure whose main ingredients are given by the transition densities of the states taken into consideration.

\section{TRANSITION DENSITIES}

\section{A. Macroscopic model for the isovector giant dipole resonance state}

In macroscopic models the collective vibrational modes are considered as oscillations of the nuclear surface and therefore they are often associated with the deformation of the density, usually described in terms of the derivative of the ground-state density. Consequently the macroscopic form factors have been mainly expressed in terms of the derivative of the optical potential which governs the elastic scattering of the colliding nuclei. These macroscopic form factors describe quite well the inelastic excitation process of most of the collective states [17-19] except for the dipole and monopole modes. In particular, for the dipole states one has to take care of the center-of-mass motion which gives rise to a spurious state and does not correspond to a real excitation of the nucleus. For this mode specific models have been formulated to take into account this aspect. The Steinwedel and Jensen [20] model as well as the Goldhaber and Teller [21] models pictured the isovector giant dipole resonance (GDR) state as an oscillation of neutrons against protons: one maintaining the nucleons within a rigid sphere (Steinwedel and Jensen) and the other allowing the rigid sphere of protons and neutrons to vibrate one against the other (Goldhaber and Teller). In the latter model the spurious c.m. motion is taken into account by scaling in a proper way the proton and neutron transition densities [22]:

$$
\delta \rho_{p}(r)=-\delta_{1} \frac{2 N}{A} \frac{d \rho_{p}}{d r}, \quad \delta \rho_{n}(r)=-\delta_{1} \frac{2 Z}{A} \frac{d \rho_{n}}{d r},
$$

where $\delta_{1}$ is the so-called deformation length and $\rho_{p}\left(\rho_{n}\right)$ are the proton (neutron) densities. If the proton and neutron densities have the same radial form, that is, their ratio is equal to $N / Z$, then the isoscalar transition density is zero. This is expected to be a good approximation for nuclei with $N=Z$; however, when $N>Z$ the proton and neutron densities may have different radial shapes producing an isoscalar transition 
density different from zero and therefore making possible an excitation of the isovector GDR by an isoscalar probe. Indeed, expanding it in the neutron excess parameter, according to Satchler [22], the isoscalar transition density for the GDR state can be written in the form

$$
\delta \rho_{\mathrm{GDR}}^{\mathrm{IS}}(r) \approx \delta_{1} \gamma\left(\frac{N-Z}{A}\right)\left[\frac{d \rho(r)}{d r}+\frac{R_{0}}{3} \frac{d^{2} \rho(r)}{d r^{2}}\right],
$$

where $R_{0}=\left(R_{n}+R_{p}\right) / 2$ is the average radius of the total nuclear density $\rho(r)$ and the parameter $\gamma$ is related to the measure of the neutron skin $\Delta R=R_{n}-R_{p}$ by the relation $\gamma(N-Z) / A=3 / 2\left(\Delta R / R_{0}\right)$ [23]. The isoscalar transition density is therefore, to leading order, directly proportional to the size of the neutron skin.

\section{B. Macroscopic model for the high-lying isoscalar dipole state}

In addition to the giant dipole resonance another collective state is expected due to $3 \hbar \omega$ nuclear transitions generated by the second-order $\Delta L=1$ transition operator and which can be seen as a compressional mode. Also for this isoscalar dipole excitation a macroscopic collective model description is possible. Such formulation has been done in Refs. [24,25] where a macroscopic collective transition density has been extracted from the isoscalar dipole sum rule. Assuming that the isoscalar dipole energy-weighted sum rule is fully exhausted by a single collective state, the corresponding isoscalar transition density, as deduced by Harakeh and Dieperink (HD) is [25]

$$
\begin{aligned}
\delta \rho^{\mathrm{HD}}(r)= & -\frac{\beta_{1}}{R \sqrt{3}}\left[3 r^{2} \frac{d}{d r}+10 r-\frac{5}{3}\left\langle r^{2}\right\rangle \frac{d}{d r}\right. \\
& \left.+\epsilon\left(r \frac{d^{2}}{d r^{2}}+4 \frac{d}{d r}\right)\right] \rho_{0}(r),
\end{aligned}
$$

where the coupling parameter $\beta_{1}$ is

$$
\beta_{1}^{2}=-\left(\frac{6 \pi \hbar^{2}}{m A E_{x}}\right) \frac{R^{2}}{\left(11\left\langle r^{4}\right\rangle-\frac{25}{3}\left\langle r^{2}\right\rangle^{2}-10 \epsilon\left\langle r^{2}\right\rangle\right)},
$$

with

$$
\epsilon=\left(\frac{4}{E_{2}}+\frac{5}{E_{0}}\right) \frac{\hbar^{2}}{3 m A},
$$

$E_{2}$ and $E_{0}$ being the giant quadrupole and giant monopole excitation energies, respectively. The distance $R$ is the half-density radius of the mass distribution. The transition density expression contains the c.m. correction, and the terms containing $\epsilon$ may be disregarded for nuclei with $A \geqslant 40$. It is straightforward to show that the condition of translational invariance $\int \delta \rho^{\mathrm{HD}}(r) r^{3} d r=0$ is satisfied.

\section{Macroscopic model for the pygmy state}

Most of the microscopic theoretical calculations have shown that the excitation of the pygmy dipole resonances is due, in the most part, to the presence of a neutron excess and this has been verified in both stable and unstable nuclei. The most popular picture behind this mode corresponds to an out of phase oscillation of a core, formed by proton and neutron, against a neutron skin which may be formed by the

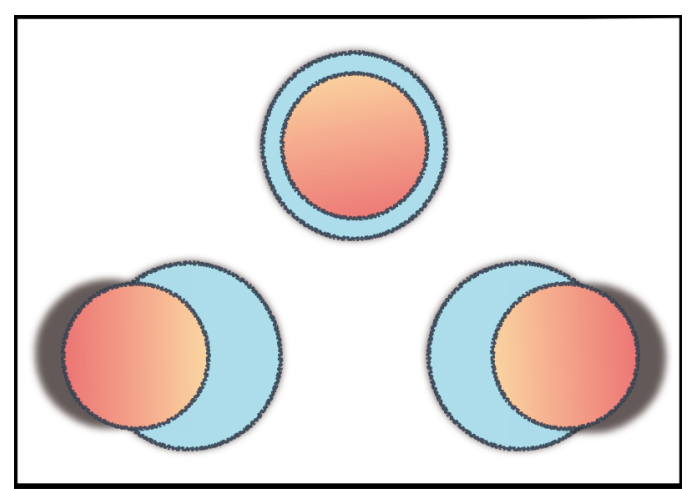

FIG. 1. (Color online) Schematic representation of the PDR state generated as an oscillation of the core against the neutron skin.

neutron excess. This can be visualized in the cartoon shown in Fig. 1 where the oscillation is schematically shown. To keep the center of mass steady, the displacement of the core and the skin, along the axis which join the center of mass of the skin and the core, should be in the form

$$
\Delta x_{S}=-x \frac{Z+N_{C}}{A}, \quad \Delta x_{C}=x \frac{N_{S}}{A},
$$

where the indices $C$ and $S$ stand for core and skin, respectively.

The density is then the sum of three terms

$$
\rho(r)=\rho_{p}(r)+\rho_{n}^{C}(r)+\rho_{n}^{S}(r),
$$

and if one assumes that the transition densities are given by a modified Goldhaber-Teller model [21]

$$
\begin{aligned}
& \delta \rho_{n}(r)=\delta\left[\frac{N_{S}}{A} \frac{d \rho_{n}^{C}(r)}{d r}-\frac{N_{C}+Z}{A} \frac{d \rho_{n}^{S}(r)}{d r}\right], \\
& \delta \rho_{p}(r)=\delta\left[\frac{N_{S}}{A} \frac{d \rho_{p}(r)}{d r}\right],
\end{aligned}
$$

one can construct the isoscalar and isovector transition densities

$$
\begin{aligned}
& \delta \rho_{\mathrm{is}}=\delta\left[\frac{N_{S}}{A} \frac{d\left(\rho_{n}^{C}+\rho_{p}\right)}{d r}-\frac{N_{C}+Z}{A} \frac{d \rho_{n}^{S}}{d r}\right], \\
& \delta \rho_{\mathrm{iv}}=\delta\left[\frac{N_{S}}{A} \frac{d\left(\rho_{n}^{C}-\rho_{p}\right)}{d r}-\frac{N_{C}+Z}{A} \frac{d \rho_{n}^{S}}{d r}\right] .
\end{aligned}
$$

The neutron core density as well as the neutron skin one can be calculated within the Hartree-Fock (HF) approach imposing that the core is formed by the protons and neutrons occupying equal orbitals. We are implicitly assuming that the protons are more strongly interacting with the neutrons of the core than with the neutrons of the skin. We can then calculate the derivatives numerically in order to get the transition densities of Eqs. (7) and (8).

\section{Microscopic model for the isoscalar dipole states}

The transition densities that we commonly use are determined with the wave function and the $X$ and $Y$ amplitudes coming from a HF plus random-phase approximation (RPA) calculation. The explicit expression can be found in Eq. (B.37) 


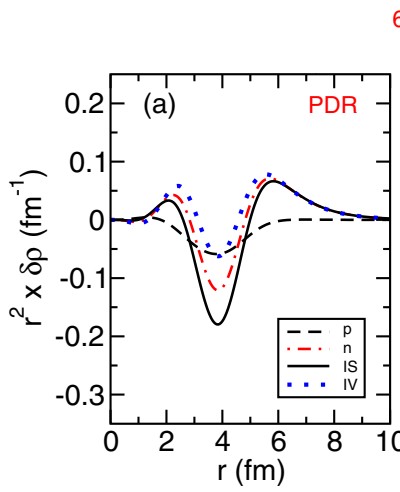

${ }^{68} \mathrm{Ni}$

FIG. 2. (Color online) RPA transition densities for the (a) lowlying dipole states and (b) ISGDR for ${ }^{68} \mathrm{Ni}$ calculated with the SGII interaction. As indicated in the legend, we plot the proton, neutron, isoscalar, and isovector components.

of Ref. [19]. Here, we present calculations done for the nucleus ${ }^{68} \mathrm{Ni}$ with a SGII interaction [26] and in Fig. 2 we show the transition densities for the PDR state (at $E=10.4 \mathrm{MeV}$ ) and the ones for the isoscalar giant dipole resonance (ISGDR), which is predicted by our RPA calculation at an excitation energy $E=32.2 \mathrm{MeV}$ [see Fig. 1(b) of Ref. [27]]. The transition densities for protons (black dashed line) and neutron (red dot-dashed line) as well the isoscalar (black solid line) and isovector (blue dotted line) combinations are shown separately in the figure. In Fig. 2(a) the trend shown is typical of the PDR states: the proton and neutron transition densities are in phase inside the nucleus while at the surface only the neutrons contribute to the isovector and isoscalar components which have the same shape and strength. Indeed, in most of the microscopic many-body calculations this state is found to be essentially a neutron particle-hole mode. In Fig. 2(b), the characteristic shapes of a compressional mode is shown by the transition densities of the ISGDR: the proton and neutron contributions are always in phase giving rise to a strong isoscalar component with a node close to the nuclear radius.

The comparison among the transition densities of the Macroscopic Pygmy Model (MPM) described by Eq. (7) with the ones calculated within the RPA model is shown in Fig. 3, where proton and neutron transition densities for the two different approaches are plotted. The HF ground-state densities were used for protons and neutrons. In this case the normalization has been done imposing that the integral of the transition density for protons (times $r^{3} d r$ ) for the MPM was equal to the corresponding RPA one. The comparison shows that the general behaviors of the transition densities are not very different; however, they differ in some details which are important for the cross-section calculations. The transition densities for protons are very similar but the one corresponding to the MPM has the peak shifted to a peripheral nuclear region. The maxima of the neutron transition densities are at the same surface region but the microscopic goes down slower then the macroscopic one. This can be ascribed to the fact that the microscopic transition densities are built up from the particle-hole configurations which may have, in particular for the neutron component, tails longer than those associated

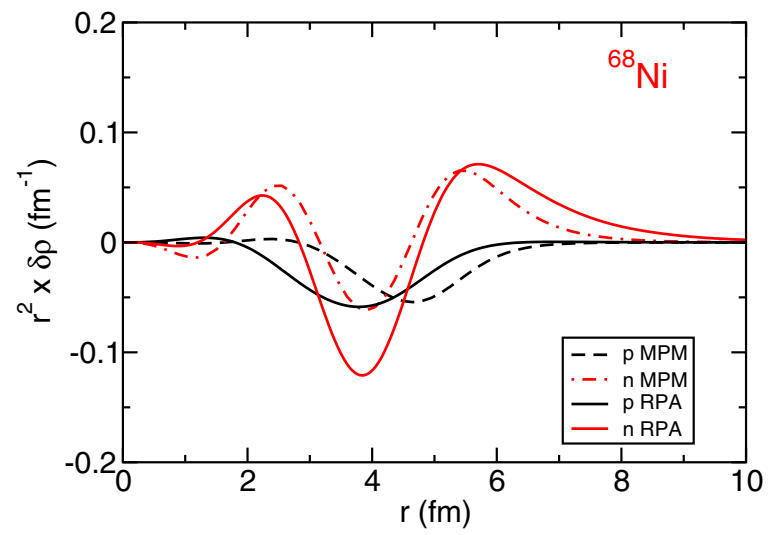

FIG. 3. (Color online) Comparison among the RPA transition densities and the ones calculated within our MPM [Eq. (7)] for the low-lying dipole states for ${ }^{68} \mathrm{Ni}$. As indicated in the legend, we plot the proton and neutron transition densities for both microscopic and macroscopic calculations.

with the occupied single-particle orbits. For the macroscopic transition densities, since they are constructed as derivative of the ground-state densities, the difference between their peaks cannot be larger then the difference of their density radii.

We move now to a comparison with the isoscalar HD model. To compare the HD transition density with the microscopic RPA one, a normalization of the $\delta \rho^{\mathrm{HD}}$ is in order. Because of the translational invariance condition the normalization is given by imposing the following equality:

$$
\int \delta \rho^{\mathrm{RPA}}(r) r^{5} d r=\int \delta \rho^{\mathrm{HD}}(r) r^{5} d r .
$$

The calculation is again done for the nucleus ${ }^{68} \mathrm{Ni}$ and in Fig. 4 the microscopic RPA isoscalar transition density is compared with $\delta \rho^{\mathrm{HD}}$ for two dipole states of ${ }^{68} \mathrm{Ni}$. The same ground-state density (HF with the SGII interaction) employed for the RPA calculations has been used to build up the $\delta \rho^{\mathrm{HD}}$. In Fig. 4(b), the transition densities for the ISGDR are shown for the two different calculations. The transition densities show the typical pattern of a compressional mode with a node in the

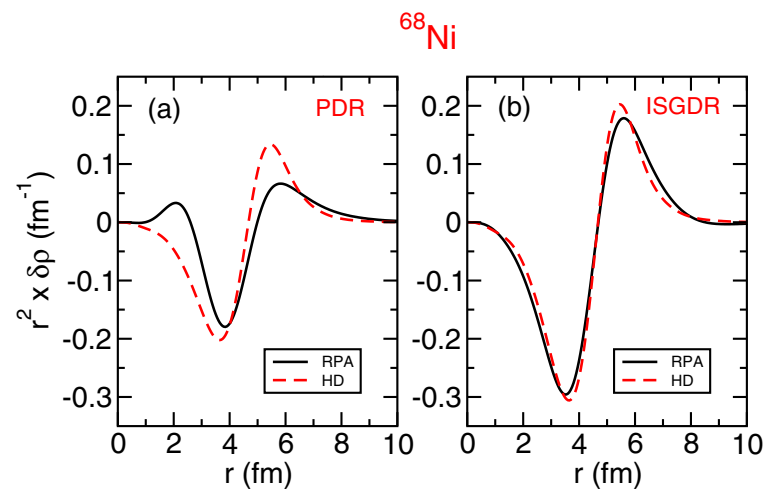

FIG. 4. (Color online) Comparison between the isoscalar microscopic (RPA) transition densities (solid black line) and the one calculated according to Eq. (3) (HD) (red dashed line) for two dipole states of ${ }^{68} \mathrm{Ni}$ ( (a) PDR state, (b) ISGDR state. 


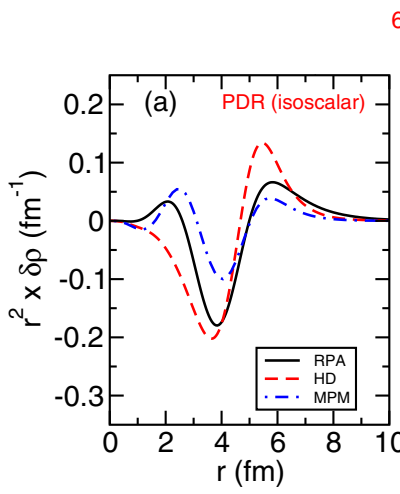

${ }^{68} \mathrm{Ni}$

FIG. 5. (Color online) Comparison among the microscopic (RPA) transition densities, one calculated according to Eq. (3) (HD), and one by the MPM, Eq. (7), for the PDR state of ${ }^{68} \mathrm{Ni}$.

interior of the nucleus. The transition densities calculated with the approach followed in Ref. [25] are almost identical, after normalization, to the RPA one. On the contrary, the agreement for the low-lying dipole state, found at $E=10.4 \mathrm{MeV}$, is not good: for $\delta \rho^{\mathrm{HD}}$ the peak at the nuclear surface is too high and it goes down a bit faster than the RPA one. The differences are due to the fact that, as it is well known and as it is shown in Fig. 2, the PDR has a strong isovector component which, by construction, is not present in the transition densities built up in Ref. [25]. So if one adopts for the PDR state the same prescription used for the ISGDR, one is implicitly assuming that the pygmy state is a pure isoscalar state which is in contradiction to what has been found in many theoretical many-body calculations.

The global comparison between the microscopic transition densities and those calculated with the macroscopic models of Eqs. (3) and (7) is shown in Fig. 5. In Fig. 5(a), the isoscalar transition density of Eq. (8a) is shown (blue dot-dashed line) together with the other two isoscalar transition densities already shown in Fig. 4. The agreement with the microscopic RPA one seems better at least in the peripheral region. In the MPM, one can also calculate the isovector transition density [Eq. (8b)] and this is compared with the RPA one in Fig. 5(a). In this case the agreement with the microscopic one is even better. So our crude, raw model seems to contain the fundamental characteristics which are of central importance in the definition of the PDR state: a mixing of isoscalar and isovector components which makes possible the population of the state via both isoscalar and isovector probes.

We are aware of the fact that other macroscopic models, much more sophisticated than the one presented here, have been developed for this low-lying state, such as the incompressible three-fluid model [28] (protons, neutrons of the core and the neutrons excess) in the same spirit of the Steinwedel-Jensen model [20]. In this model a low-lying dipole state is found as an oscillation of the neutrons and protons of the core against the neutrons excess, although the calculated strength was too weak. A modified version of this model is given in Ref. [29] where only two incompressible fluids were considered, namely, the core and the neutron excess. The strength of the low-lying dipole state coming out is in reasonable agreement with the experimental data available at that time [30], for some $\beta$ stable nuclei having few neutron excess. Another macroscopic approach that takes explicitly into account the neutron skin [31] follows the Goldhaber-Teller prescription [21], finding results similar to the ones obtained in Ref. [29]. Another macroscopic model where the nucleus is considered as a spherical piece of elastic continuous medium (static core) [32] has investigated the PDR in terms of an elastodynamic excitation mechanism of peripheral dynamic layers implying the pure isoscalar nature of the state.

\section{NUCLEAR FORM FACTORS}

The description of inelastic nuclear excitation can be done within different approaches ranging from the first-order theory, like DWBA where elastic scattering is dominant, to the coupled-channel models where higher order effects, like the strong coupling to other states outside the one taken in consideration, are explicitly worked out. Along with these full quantal calculations, semiclassical models have been developed and used for the description of heavy-ion collision and inelastic cross-section calculations. In all these approaches, one of the most important ingredients is the determination of the form factors.

The expression of the form factors can be derived either within a macroscopic collective model or in a microscopic approach. One of the most effective ways to construct a microscopic form factor is by using the well-known doublefolding procedure $[17,33]$. In this method the double-folding potential between two heavy ions is obtained by integrating the nucleon-nucleon interaction over the densities of the two nuclei. In a similar way the form factors are constructed by using the density of one nucleus on one side and the transition densities of the exited nucleus on the other side.

In previous work $[8,9,34]$ we have followed this procedure and the internal structure of the nucleus has been described by means of a many-body model (often HF plus RPA calculations were used) which yielded microscopic neutron and proton transition densities. In addition one should include the isospindependent part of the nucleon-nucleon interaction

$$
v_{12}=v_{0}\left(r_{12}\right)+v_{1}\left(r_{12}\right) \boldsymbol{\tau}_{1} \cdot \boldsymbol{\tau}_{2},
$$

where the isoscalar part $v_{0}$ generates an isoscalar ion-ion potential and the isovector term $v_{1}$ gives an isospin-dependent folding potential; here the $\boldsymbol{\tau}_{i}$ 's are the isospins of the nucleons. With this choice the form factors have two components with the following forms:

$$
\begin{aligned}
F_{0}= & \iint\left[\delta \rho_{n}^{A}\left(r_{1}\right)+\delta \rho_{p}^{A}\left(r_{1}\right)\right] \\
& \times v_{0}\left(r_{12}\right)\left[\rho_{p}^{B}\left(r_{2}\right)+\rho_{n}^{B}\left(r_{2}\right)\right] r_{1}^{2} d r_{1} r_{2}^{2} d r_{2}, \\
F_{1}= & \iint\left[\delta \rho_{n}^{A}\left(r_{1}\right)-\delta \rho_{p}^{A}\left(r_{1}\right)\right] \\
& \times v_{1}\left(r_{12}\right)\left[\rho_{n}^{B}\left(r_{2}\right)-\rho_{p}^{B}\left(r_{2}\right)\right] r_{1}^{2} d r_{1} r_{2}^{2} d r_{2} .
\end{aligned}
$$

where the $\delta \rho_{i}(i=n, p)$ are the transition densities of the state under study of nucleus $A$ which is excited by the mean field of 


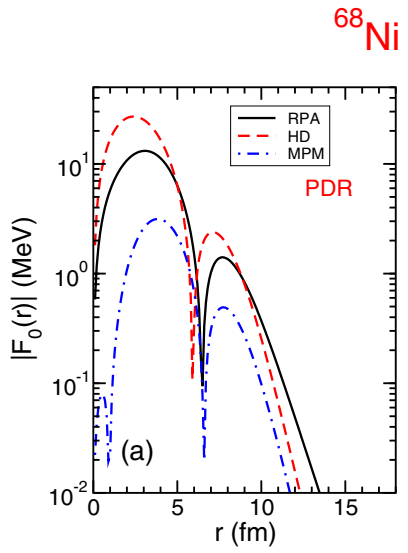

${ }^{68} \mathrm{Ni}+{ }^{12} \mathrm{C}$

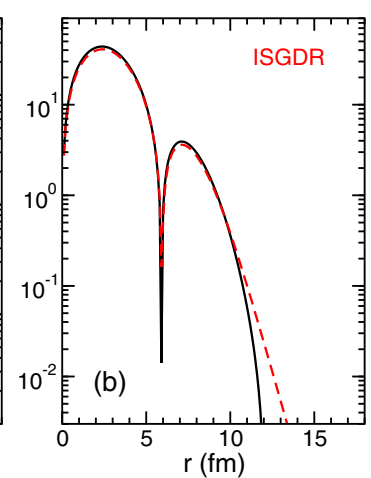

FIG. 6. (Color online) Form factors for the system ${ }^{68} \mathrm{Ni}+{ }^{12} \mathrm{C}$ for the PDR state left frame and for the ISGDR right frame. All of them are calculated with the double-folding procedure but starting from different transition densities: microscopic RPA, HD, and MPM [Eq. (7)].

nucleus $B$. In the particular case when $\rho_{n} / \rho_{p}=(N / Z)$, then the form factor (12) is zero when one or both partners of the reaction have $N=Z$ [17]. For $v_{0}$ and $v_{1}$ we use the M3Y nucleon-nucleon interaction, Reid type [35], whose explicit expressions can be found in Ref. [33]. This interaction is not density dependent and it has been successfully used for the description of several cases of elastic and inelastic reactions. The implementation of a density dependence shows that the obtained potential are almost not distinguishable. However, when different density dependences are taken into account there are some differences among the corresponding potential obtained. However, these differences are in the interior part of the nucleus while at the surface the potentials are practically identical [36]. Therefore, since the reactions studied with this model explore the peripheral region of the two colliding nuclei, the use of the M3Y, as we employ it, seems to be well justified.

The calculation of the form factors are done in the momentum-space making use of the Fourier transform because the calculation with this method is fast and convenient [33].

With the transition densities constructed as described in the previous section we can calculate the corresponding form factors. This has been done for the system ${ }^{68} \mathrm{Ni}+{ }^{12} \mathrm{C}$ which will be the subject of an experimental investigation in the near future. The nuclear form factors have been calculated with the double-folding procedure with the three transition densities described above and the results are shown in Fig. 6 for the two dipole states under study.

For the ISGDR state, the microscopic nuclear form factor (solid black line) and the one calculated with the Harakeh and Dieperink (HD) transition densities (red dashed line) are almost identical in all the significant distance range as can be seen in Fig. 6(b). Only in the very peripheral region can one appreciate some difference and this almost certainly will not have any effect when used for cross-section calculations.

On the contrary, strong differences can be appreciated in the comparisons in the PDR state. In Fig. 6(a), the red dashed line, corresponding to the HD form factor, is entirely different from the microscopic one (black solid line) both in the intensity and especially in the slope in the most important surface region. Also for the form factor calculated with the transition density of the MPM the agreement is not at all satisfactory, although the position of the second maximum is the same as the microscopic one. This may be surprising since, at first glance, it seems that our isoscalar macroscopic transition density was closer to the microscopic one than the HD one (see Fig. 5). These differences have an implication on the differential cross section.

\section{INELASTIC CROSS SECTION}

Once the form factors are constructed, the inelastic cross section can be calculated within the full quantal coupledchannel model or within its semiclassical version, among others. To appreciate and single out the differences in the form factors calculated in the previous section we have chosen to perform the inelastic cross section calculations with the DWBA approach. We have done DWBA calculations for the system ${ }^{68} \mathrm{Ni}+{ }^{12} \mathrm{C}$ at $10 \mathrm{MeV} / \mathrm{u}$ with the code DWUCK4 [37].

As it was expected from the shape of the form factors, the differential cross section for the ISDR state, shown in Fig. 7(b), for the microscopic form factors and the HD ones are almost indistinguishable. This result shows, once again, that this state is a pure isoscalar state and it is well described by the procedure employed in Refs. [24,25]. On the other hand, the agreement for the PDR case is not good: the results corresponding to the HD form factors are higher with respect to the microscopic one up to a factor of 3 in the more important forward angular region. Also for our macroscopic pygmy model the results are far from the microscopic ones with the difference that they differ by the same factor in the whole angular region. This can be ascribed to the different transition density shapes at the surface of the nucleus. The distinct slopes in the surface region (see Fig. 3) give rise to different slopes in the same region of the form factors. This is the part which gives the stronger contribution to the excitation process since we are dealing with surface processes. Indeed, the almost factor of 3 in the external region of the form factors is generating a factor of almost 9 in the cross section.

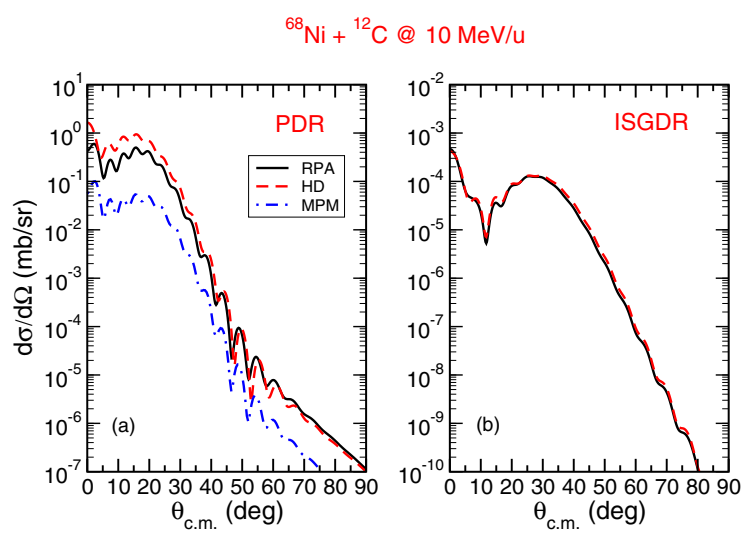

FIG. 7. (Color online) Differential cross sections for the system ${ }^{68} \mathrm{Ni}+{ }^{12} \mathrm{C}$ at $10 \mathrm{MeV} / \mathrm{u}$ for the (a) PDR state and (b) ISGDR state. They have been calculated with the DWUCK 4 code. 
Our microscopic form factor has been successfully employed in the analysis of various experiments performed with

${ }^{17} \mathrm{O}$ as isoscalar probe [14-16]. In these papers, only the use of the microscopic form factor has allowed the extraction of the isoscalar components of the $1^{-}$excited states.

\section{CONCLUSIONS}

The low-lying dipole state (PDR) is a mode whose isoscalar and isovector components are strongly mixed as it is clearly manifested in their transition densities. This feature opens the possibility of studying these PDR states by using an isoscalar probe in addition to the conventional isovector one. Recently, many experiments have been performed using isoscalar probes such as $\alpha$ particles [2,10-13] or ${ }^{17} \mathrm{O}$ [14-16] and, according to the standard prescription, these experiments have been analyzed by comparing the experimental differential cross section with theoretical calculations. The latter rely very much on the radial form factors which, in the standard codes, are constructed according to collective model prescriptions.

Here we have compared results of differential cross-section calculations based on three different structure models, using in all cases the double-folding procedure to generate the form factors from the transition densities. The transition densities used were the one extracted by a sum rule approach deduced for the high-lying ISGDR [24,25], the one deduced according to a simple macroscopic model for the PDR, and those obtained from a microscopic RPA approach. The comparison among the models has shown a very good agreement with the RPA for the high-lying ISGDR state for both the form factors as well as for the differential cross section. In the case of the PDR state, on the other hand, the weakly bound nature of the involved orbitals leads to different slopes in the tails of the macroscopic and microscopic transition densities, with consequent differences in the corresponding form factors and cross sections.

Therefore, our message is that one has to carefully use the proper form factor in the experimental analysis of PDR state excitations. According to the standard procedure, a DWBA calculation based on collective models is employed to establish the amount of $B(E 1)$ to attribute to the state as well as the percentage of EWSR to be assigned to it. Since we have shown that the use of different form factors may lead to different differential cross sections, it is of paramount importance to use the "correct" microscopically based one. Use of inappropriate form factors has consequences on the calculated cross section and therefore in jeopardizing the extracted physical quantities. Only the microscopic form factor, by construction, includes all the important properties of these states, starting from the strong mixing of isoscalar and isovector characters.

\section{ACKNOWLEDGMENTS}

M.V.A. acknowledges the financial support provided by the Ministerio de Economía y Competitividad (FIS2013-41994P, FIS2011-28738-c02-01), and by Consejería Economía, Innovación, Ciencia y Empleo, Junta de Andalucía (FQM-160, P11-FQM-7632).
[1] N. Paar, D. Vretenar, E. Khan, and G. Colò, Rep. Prog. Phys. 70, 691 (2007) and referencs therein.

[2] D. Savran, T. Aumann, and A. Zilges, Prog. Part. Nucl. Phys. 70, 210 (2013) and references therein.

[3] A. Klimkiewicz et al. (LAND Collaboration), Phys. Rev. C 76, 051603(R) (2007).

[4] P. G. Reinhard and W. Nazarewicz, Phys. Rev. C 81, 051303 (2010).

[5] A. Tamii et al., Phys. Rev. Lett. 107, 062502 (2011).

[6] J. Piekarewicz, B. K. Agrawal, G. Colò, W. Nazarewicz, N. Paar, P.-G. Reinhard, X. Roca-Maza, and D. Vretenar, Phys. Rev. C 85, 041302 (2012).

[7] A. Vitturi, E. G. Lanza, M. V. Andrés, F. Catara, and D. Gambacurta, PRAMANA 75, 73 (2010); J. Phys.: Conf. Ser. 267, 012006 (2011).

[8] E. G. Lanza, F. Catara, D. Gambacurta, M. V. Andrés, and Ph. Chomaz, Phys. Rev. C 79, 054615 (2009).

[9] E. G. Lanza, A. Vitturi, M. V. Andrés, F. Catara, and D. Gambacurta, Phys. Rev. C 84, 064602 (2011).

[10] D. Savran, M. Babilon, A. M. van den Berg, M. N. Harakeh, J. Hasper, A. Matic, H. J. Wörtche, and A. Zilges, Phys. Rev. Lett. 97, 172502 (2006).

[11] J. Endres D. Savran, A. M. van den Berg, P. Dendooven, M. Fritzsche, M. N. Harakeh, J. Hasper, H. J. Wörtche, and A. Zilges, Phys. Rev. C 80, 034302 (2009).

[12] J. Endres et al., Phys. Rev. Lett. 105, 212503 (2010).

[13] J. Endres et al., Phys. Rev. C 85, 064331 (2012).
[14] F. C. L. Crespi et al., Phys. Rev. Lett. 113, 012501 (2014)

[15] L. Pellegri et al., Phys. Lett. B 738, 519 (2014).

[16] F. C. L. Crespi et al., Phys. Rev. C 91, 024323 (2015).

[17] G. R. Satchler, Direct Nuclear Reactions (Oxford University, Oxford, 1983).

[18] R. A. Broglia and Aa. Winther, Heavy-ion Reactions (AddisonWesley, Reading, MA, 1991).

[19] S. Landowne and A. Vitturi, in Treatise on Heavy-Ion Science, edited by D. A. Bromley (Plenum, New York, 1984), Vol. 1, p. 355 .

[20] H. Steinwedel and J. H. D. Jensen, Z. Naturforsch. 5a, 413 (1950).

[21] M. Goldhaber and E. Teller, Phys. Rev. 74, 1046 (1948).

[22] G. R. Satchler, Nucl. Phys. A 472, 215 (1987); S. Shlomo, Y.-W. Lui, D. H. Youngblood, T. Udagawa, and T. Tamura, Phys. Rev. C 36, 1317 (1987); K. Nakayama and G. Bertsch, Phys. Rev. Lett. 59, 1053 (1987).

[23] F. Catara, E. G. Lanza, M. A. Nagarajan, and A. Vitturi, Nucl. Phys. A 624, 449 (1997).

[24] T. J. Deal, Nucl. Phys. A 217, 210 (1973).

[25] M. N. Harakeh and A. E. L. Dieperink, Phys. Rev. C 23, 2329 (1981).

[26] N. Van Giai and H. Sagawa, Phys. Lett. B 106, 379 (1981); Nucl. Phys. A 371, 1 (1981).

[27] E. G. Lanza, A. Vitturi, and M. V. Andrés, J. Phys.: Conf. Ser. 420, 012147 (2013). 
[28] R. Mohen, M. Danos, and L. C. Biedenharn, Phys. Rev. C 3, 1740 (1971).

[29] Y. Suzuki, K. Ikeda, and H. Sato, Prog. Theor. Phys. 83, 180 (1990).

[30] M. Igashira, H. Kitazawa, M. Shimizu, H. Komano, and N. Yamamuro, Nucl. Phys. A 457, 301 (1986).

[31] P. Van Isacker, M. A. Nagarajan, and D. D. Warner, Phys. Rev. C 45, R13 (1992).

[32] S. I. Bastrukov, I. V. Molodtsova, D. V. Podgainy, Ş. Mişicu and H.-K. Chang, Phys. Lett. B 664, 258 (2008).
[33] G. R. Satchler and W. G. Love, Phys. Rep. 55, 183 (1979).

[34] E. G. Lanza, F. Catara, M. V. Andrés, Ph. Chomaz, M. Fallot and J. A. Scarpaci, Phys. Rev. C 74, 064614 (2006).

[35] G. Bertsch, J. Horysowicz, H. McManus, and W. G. Love, Nucl. Phys. A 284, 399 (1977).

[36] Dao T. Khoa and W. von Oertzen, Phys. Lett. B 342, 6 (1995); Dao T. Khoa, G. R. Satchler, and W. von Oertzen, Phys. Rev. C 56, 954 (1997)

[37] P. D. Kunz, DWUCK4 code for DWBA at http://spot.colorado. edu/ kunz/DWBA.html 